\title{
QUASICONFORMAL MAPPINGS WITHOUT BOUNDARY EXTENSIONS
}

\section{T. KUUSALO}

1. According to the classical theorem of C. Carathéodory [1] and of W. F. Osgood and E. H. Taylor [6], a conformal mapping $f: D \rightarrow D^{\prime}$ between two Jordan domains $D$ and $D^{\prime}$ in the complex plane $C$ always has a boundary extension, i.e. there is a homeomorphism $f^{*}: \bar{D} \rightarrow \bar{D}^{\prime}$ of the respective closed domains such that $\left.f^{*}\right|_{D}=f$. As it is well known, the theorem holds for planar quasiconformal mappings as well. In fact, G. Faber's proof for conformal mappings carries over to the quasiconformal case with minor modifications only ([3], cf. also R. Courant [2]; LehtoVirtanen [5], pp. 44-46). J. Väisälä proved in [7] the existence of a boundary extension for all quasiconformal mappings $f: D \rightarrow D^{\prime}$ between $n$-dimensional Jordan domains $D, D^{\prime} \subset \boldsymbol{R}^{n}$ quasiconformally equivalent to the unit ball $B^{n} \subset \boldsymbol{R}^{n}, n \geqq 2$ (also in Väisälä [8], pp. 51-67). But unlike the planar case, even a Jordan domain $D \subset \boldsymbol{R}^{n}$ homeomorphic to the unit ball $B^{n}$ is not necessarily quasiconformally equivalent to $B^{n}$ when $n \geqq 3$ (Gehring-Väisälä [4]). We shall give below an example which shows that for arbitrary Jordan domains $D, D^{\prime} \subset R^{n}, n \geqq 3$, a quasiconformal mapping $f: D \rightarrow D^{\prime}$ does not always have a boundary extension.

I wish to express here my gratitude to Vesa Lappalainen, who has made all the excellent drawings below using the computer facilities of the University of Jyväskylä. Furthermore, I would like to thank the Royal Swedish Academy of Sciences for supporting my stay at the Mittag-Leffler Institute during the academic year $1983-1984$.

2. Let $\Gamma$ be the spiral curve in the closed unit disc $\bar{B}^{2} \subset C$ described by

$$
\Gamma(r)=r e^{(i \alpha / 2)(r-r-1)}
$$

for $0 \leqq r \leqq 1, \alpha>0$ a constant. If $\varrho=\varrho(z)=\varrho(z, 0)$ is the hyperbolic distance of $z \in \bar{B}^{2}$ from the origin with respect to the hyperbolic length element

$$
d \varrho=2\left(1-|z|^{2}\right)^{-1}|d z|,
$$

and $\theta=\arg (z)$ the argument of $z$, the trace of $\Gamma$ can also be given as

$$
\Gamma=\left\{z \in \bar{B}^{2}: \theta=-\frac{\alpha}{\sinh (\varrho)}, \quad 0 \leqq \varrho \leqq+\infty\right\} .
$$


The curve $\Gamma$ has hyperbolic curvature

$$
\chi(\varrho)=\frac{1+\alpha^{-2} \tanh ^{4}(\varrho)}{\tanh (\varrho)\left[1+\alpha^{-2} \tanh ^{2}(\varrho)\right]^{3 / 2}}
$$

at the parameter value $r=\tanh (\varrho / 2)$. Now the boundary of the domain

$$
G=\left\{z \in C: 0<|z|<1,\left|\theta+\frac{\alpha}{\sinh (\varrho)}\right|<\frac{\pi}{2}\right\}
$$

consists of a circular arc together with two spirals $\Gamma_{ \pm}= \pm i \Gamma$ (cf. Figure 1; here as well as in all subsequent drawings $\alpha=2$ ). Because the closed domain $\bar{G}$ lies to the right of the boundary arc $\Gamma_{+}$, and the hyperbolic curvature of $\Gamma_{+}$is positive, we can define in $\bar{G}$ a hyperbolic orthogonal projection $p: \bar{G} \rightarrow \Gamma_{+}$such that for all $z \in \bar{G}$ the hyperbolic arc $z p(z) \subset \bar{G}$ is perpendicular to $\Gamma_{+}$and that $\varrho(z, p(z))$ is the hyperbolic distance of $z$ from $\Gamma_{+}$,

$$
\varrho(z, p(z))=\varrho\left(z, \Gamma_{+}\right)=\min _{z^{\prime} \in \Gamma_{+}} \varrho\left(z, z^{\prime}\right) .
$$

The projection $p$ is easily seen to be infinitely differentiable in $G$. Furthermore, the positive curvature of $\Gamma_{+}$implies that $p$ is locally strictly contractive in $G$,

$$
\varrho\left(p(z), p\left(z_{0}\right)\right)<\varrho\left(z, z_{0}\right)
$$

for all $z \neq z_{0}$ in a sufficiently small neighbourhood of $z_{0} \in G$.

If $T(\varrho)$ is a primitive of

$$
\left(1+\alpha^{2} \tanh ^{-2}(\varrho)\right)^{1 / 2},
$$

we can define by $\tau(z)=T(\varrho(z)), z \in \Gamma_{+}$, a hyperbolic length scale on $\Gamma_{+}$such that

$$
\varrho_{+}\left(z_{1}, z_{2}\right)=\left|\tau\left(z_{1}\right)-\tau\left(z_{2}\right)\right|
$$

gives the hyperbolic length of the subarc of $\Gamma_{+}$with endpoints $z_{1}, z_{2} \in \Gamma_{+}$. As $\tau(0)=-\infty, \tau(i)=+\infty$, the spiral $\Gamma_{+}$is infinitely long in both directions. We extend $\tau$ to a function $\tau: \bar{G} \rightarrow \overline{\boldsymbol{R}}$ by setting

for arbitrary $z \in \bar{G}$.

$$
\tau(z)=\tau(p(z))
$$

3. With respect to the (quasi) hyperbolic length element

$$
d \sigma=\frac{d s}{r}, \quad r=\left(x_{1}^{2}+x_{2}^{2}\right)^{1 / 2}
$$

in $\overline{\boldsymbol{R}}^{3}=\boldsymbol{R}^{3} \cup\{\infty\}$ the $x_{3}$-axis forms the circle at infinity, and a hyperbolic isometry identifying the closed unit disc $\bar{B}^{2} \subset C$ with the closed half-plane $\left\{x \in \overline{\boldsymbol{R}}^{3}: x_{2}=0, x_{1} \geqq 0\right\}$ can be defined by

$$
x_{1}+i x_{3}=h(z)=\frac{1-z}{1+z} .
$$

If we rotate the image $h(\bar{G})$ of $\bar{G}$ around the $x_{3}$-axis, we get a closed Jordan domain 
(a sectorial cross cut in Figure 2)

where:

$$
\bar{D}=\left\{x \in R^{3}:\left|\psi+\frac{\alpha}{\sinh (\sigma)}\right| \leqq \frac{\pi}{2}, \quad 0 \leqq \sigma \leqq+\infty\right\},
$$

and

$$
\psi=\psi(x)=\arg \left(\frac{1-r-i x_{3}}{1+r+i x_{3}}\right)
$$

$$
\sigma=\sigma(x)=\sigma\left(x, S^{1}\right)
$$

is the hyperbolic distance of $x \in \boldsymbol{R}^{3}$ from the unit circle $S^{1}$ of the plane $\boldsymbol{R}^{2}=$ $\left\{x \in \boldsymbol{R}^{3}: x_{3}=0\right\}$. The boundary of $\bar{D}$ consists of two topological discs

$$
E_{ \pm}=\left\{x \in \boldsymbol{R}^{3}: \psi+\frac{\alpha}{\sinh (\sigma)}= \pm \frac{\pi}{2}, \quad 0 \leqq \sigma \leqq+\infty\right\}
$$

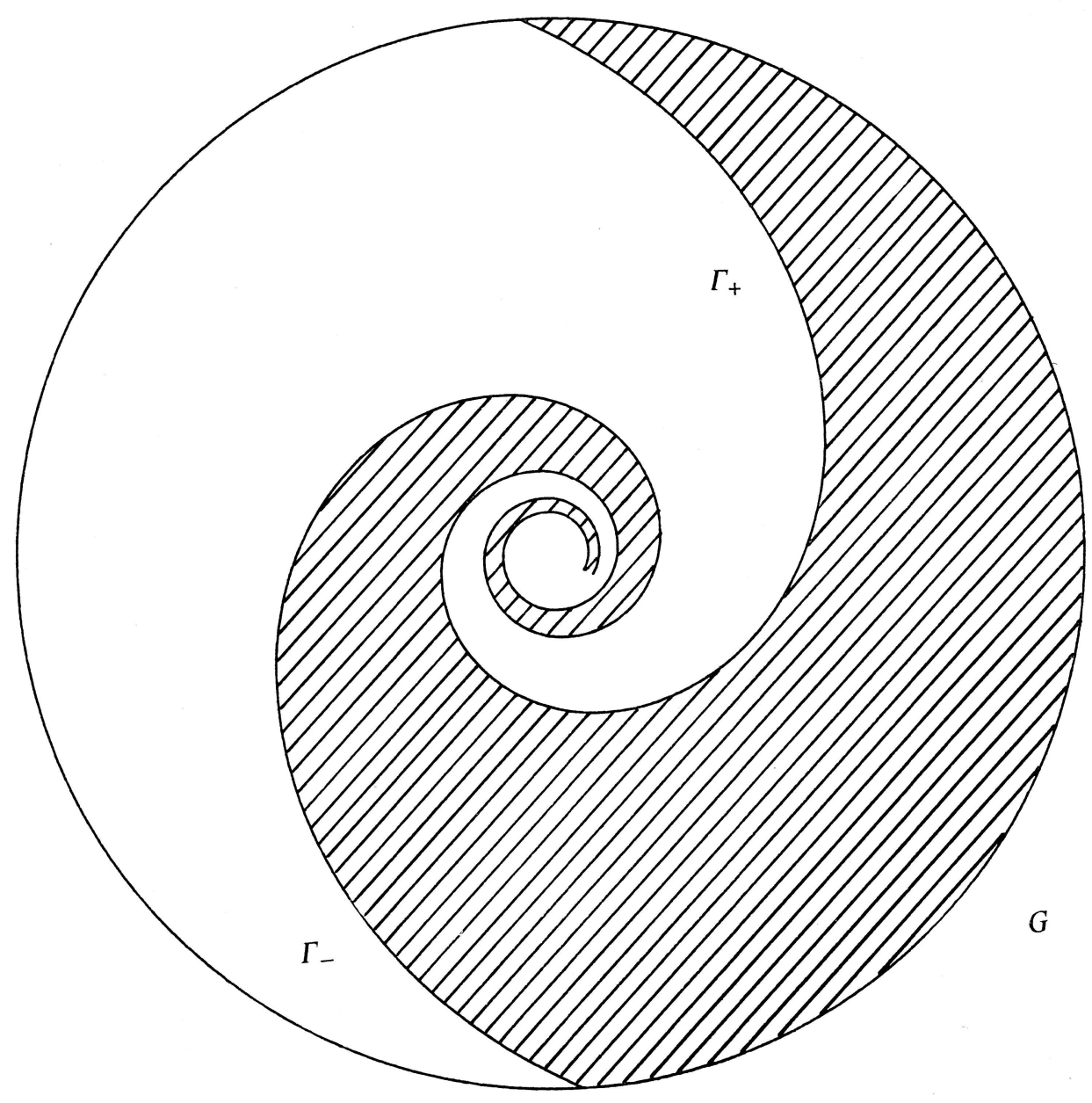

Figure 1 
glued together along the circle $S^{1}=E_{+} \cap E_{-}$. The function $\tau$ extends by rotation to a parameter function $\tau: \bar{D} \rightarrow \overline{\boldsymbol{R}}$ such that $\tau^{-1}(-\infty)=S^{1}$ and $\tau^{-1}(+\infty)=\{(0,0,-1)\}$. As in the preceding paragraph, we can define in the whole domain $\bar{D}$ outside the $x_{3}$-axis a locally contractive hyperbolic orthogonal projection $p$ into the lower halfboundary $E_{+}$such that $\tau(x)=\tau(p(x))$ for all $x$ in $\bar{D}$ outside the $x_{3}$-axis.

If we let $S^{1} \subset E_{+}$collapse to a point and set

for $x \in \boldsymbol{R}^{3}$, we see that

$$
\varphi(x)=\arg \left(x_{1}+i x_{2}\right)
$$

$$
\theta(x)=e^{\tau(x)+i \varphi(x)}
$$

defines an isometry and thus a conformal mapping of $E_{+}$onto the Riemann sphere $\bar{C}$ with respect to the hyperbolic length element $d \sigma$ in $E_{+}$and the logarithmic length element

$$
d v=|z|^{-1}|d z|
$$

in $\overline{\boldsymbol{C}}$, so that $\theta\left(S^{1}\right)=0$ and $\theta(0,0,-1)=\infty$.

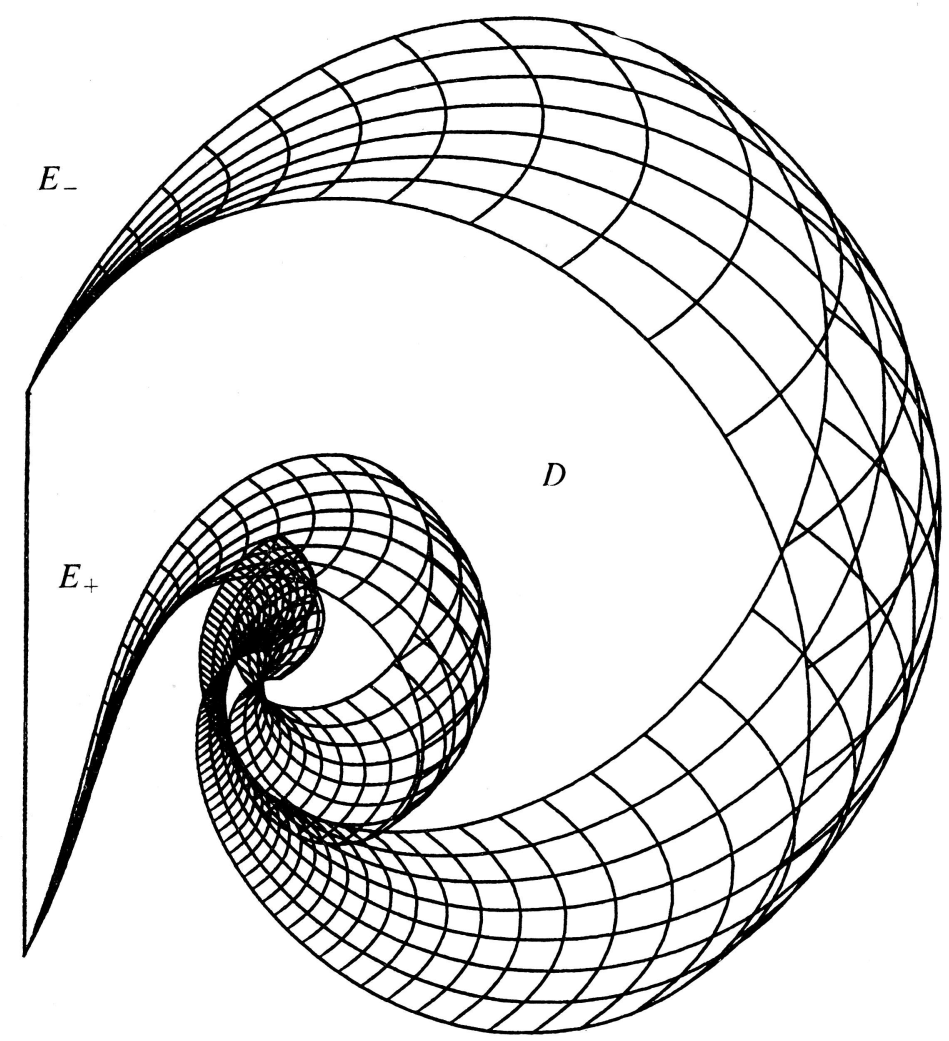

Figure 2 
Using the complex notation

$$
\begin{gathered}
x=\left(\zeta, x_{3}\right)=\left(x_{1}+i x_{2}, x_{3}\right) \in \boldsymbol{R}^{3}, \\
a x=\left(a \zeta,|a| x_{3}\right) \in \boldsymbol{R}^{3}
\end{gathered}
$$

for $x \in \boldsymbol{R}^{3}, a \in \boldsymbol{C}$, we define for any real $\lambda$ the mapping $f_{\lambda}: \bar{D} \backslash S^{1} \rightarrow \bar{D} \backslash S^{1}$ by

$$
f_{\lambda}(x)=\exp (i \lambda \tau(x)) x .
$$

The restriction $f_{\lambda}: E_{+} \backslash S^{1} \rightarrow E_{+} \backslash S^{1}$ is quasiconformal for all $\lambda \in R$, its symmetrized derivative having the eigenvalues

$$
\begin{gathered}
c_{1}(\lambda)=\left(1+\lambda^{2} / 2+\left(\lambda^{2}+\lambda^{4} / 4\right)^{1 / 2}\right)^{1 / 2}, \\
c_{2}(\lambda)=c_{1}(\lambda)^{-1}
\end{gathered}
$$

at all nonsingular points of $E_{+}$. Now $f_{\lambda}$ preserves the equidistance surfaces $S_{t}=$ $\left\{x \in D: \sigma\left(x, E_{+}\right)=t\right\}, t>0$, in $D$, and because the projection $p$ is locally contractive, we see, using appropriate local coordinates, that the eigenvalues $c_{1}(x, \lambda) \geqq c_{2}(x, \lambda) \geqq$ $c_{3}(x, \lambda)>0$ of the symmetrized derivative of $f_{\lambda}$ satisfy

$$
\begin{gathered}
c_{1}(x, \lambda)=c_{3}(x, \lambda)^{-1} \leqq c_{1}(\lambda), \\
c_{2}(x, \lambda)=1
\end{gathered}
$$

at all points $x$ of $D$ outside the $x_{3}$-axis. Thus $f_{\lambda}: D \rightarrow D$ is quasiconformal with dilatations

$$
K\left(f_{\lambda}\right)=K_{o}\left(f_{\lambda}\right)=K_{I}\left(f_{\lambda}\right)=c_{1}(\lambda)^{3} .
$$

Because $\tau(x) \rightarrow-\infty$ as $x \rightarrow S^{1}$, we have

$$
C\left(f_{\lambda}, b\right)=S^{1}
$$

for the cluster set at any boundary point $b \in S^{1} \subset \partial D$ when $\lambda \neq 0$. Thus in this case the quasiconformal mapping $f_{\lambda}: D \rightarrow D$ does not have any boundary extension. To visualize the situation we have depicted in Figure 3 the image of $\Gamma^{\prime}=h(\Gamma) \cap D$ when $\lambda=0.25$.

We could also choose a function $\lambda(\tau)$ with a uniformly bounded derivative $\lambda^{\prime}(\tau)$ converging to 0 when $\tau \rightarrow-\infty$ but such that $\lambda(\tau) \rightarrow-\infty$ at the same time, and define $f: D \rightarrow D$ by setting

$$
f(x)=\exp [i(\lambda \circ \tau)(x)] x
$$

for all $x \in D$. Then also $f: D \rightarrow D$ would be a quasiconformal mapping having $C(f, b)=S^{1}$ as cluster set at all $b \in S^{1}$ but with the local maximal dilatation $K(x, f)$ approaching one when $x$ approaches $S^{1}$.

4. Remarks. (i) The example above was chosen because it allows a fairly simple and explicit representation. The Jordan domain $D$ has also quite nice symmetry properties, e.g. the Möbius transformation generated by the successive reflections in the plane $\boldsymbol{R}^{2}$ and in the unit sphere $S^{2} \subset \boldsymbol{R}^{3}$ maps $D$ conformally onto its exterior. The boundary $\partial D=E_{+} \cup E_{-}$is quasiconformally collared with the exception of the 


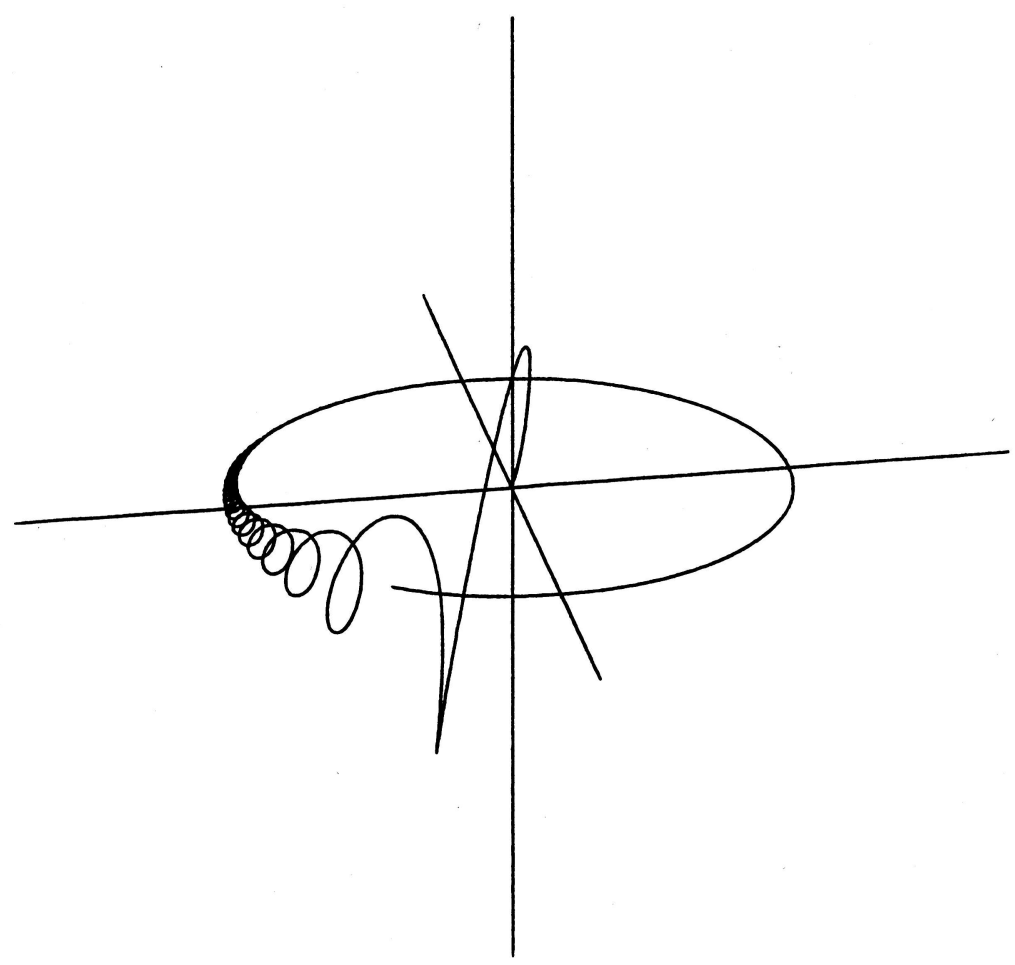

Figure 3

circle $S^{1}=E_{+} \cap E_{-}$. In fact, the mapping $\Psi$ defined by

$$
\Psi(x)=\left(\frac{e^{\tau(x)} \zeta}{|\zeta| \cosh \sigma\left(x, E_{+}\right)},-e^{\tau(x)} \tanh \sigma\left(x, E_{+}\right)\right) \in \boldsymbol{R}^{3}
$$

for $x=\left(\zeta, x_{3}\right) \in \bar{D}$ maps $D$ homeomorphically onto a domain $D^{*}$ in the lower halfspace $x_{3}<0$ bounded by the plane $\overline{\boldsymbol{R}}^{2}=\Psi\left(E_{+}\right)$and by the quasiconformal sphere $E_{-}^{*}=\Psi\left(E_{-}\right)$, the singular part $S^{1}=E_{+} \cap E_{-}$of the boundary collapsing onto the origin, $\Psi\left(S^{1}\right)=\{0\}$ (cf. Figure 4). The mapping $\Psi$ preserves $\varphi(x)=\arg (\zeta)$, and $\sigma\left(\Psi(x), R^{2}\right)=\sigma\left(x, E_{+}\right)$for all $x \in D$. In the direction of the line $\varphi(x)=\varphi\left(x_{0}\right)$, $\sigma\left(x, E_{+}\right)=\sigma\left(x_{0}, E_{+}\right)$the mapping $\Psi$ has at $x_{0} \in D$ the contraction coefficient

$$
\chi\left(x_{0}\right)=\left[1+\chi\left(\sigma\left(p\left(x_{0}\right)\right)\right) \tanh \sigma\left(x_{0}, E_{+}\right)\right]^{-1} .
$$

Because we have $\sigma\left(x, E_{-}\right) \sim \alpha^{-1} \pi \sigma^{2}$ for the width of $D$ and $\chi(\sigma) \sim \sigma^{-1}$ for the hyperbolic curvature when $x$ approaches $S^{1}$ on $E_{+}$, we see that $x$ is uniformly bounded away from 0 in $D$, so that also the mapping $\Psi: D \rightarrow D^{*}$ is quasiconformal. When $\alpha=2, \quad x(x)>1 / 3$ in the whole domain $D$, so that in this case $K(\Psi)<9$. 


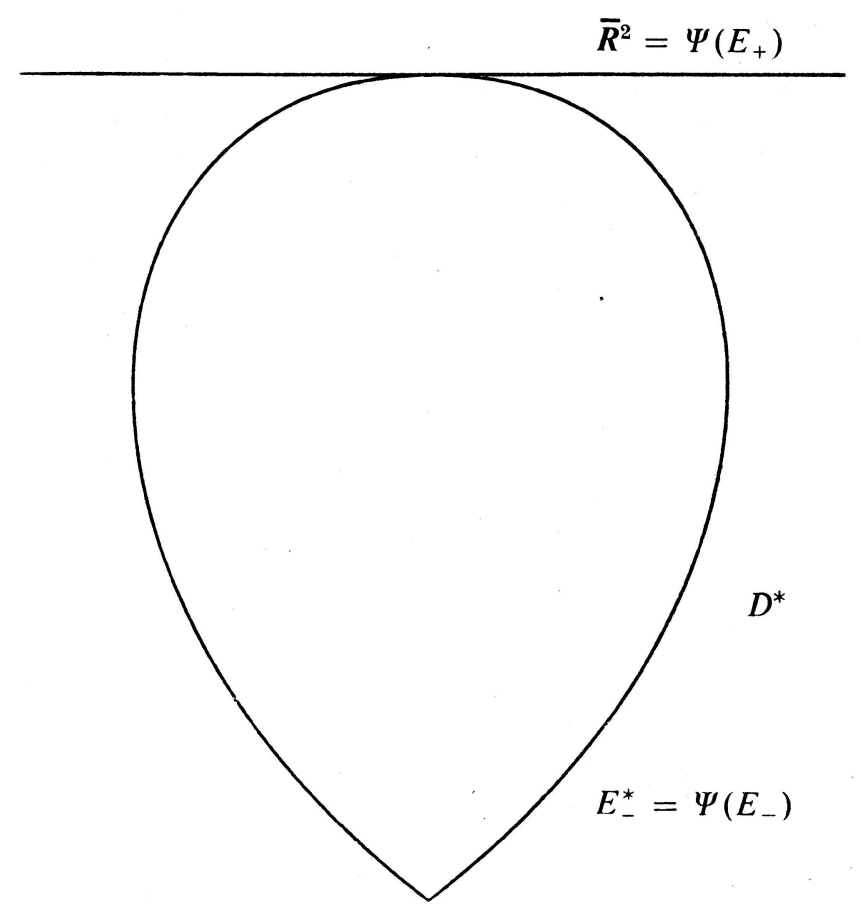

Figure 4

(ii) We can make the same construction in $\boldsymbol{R}^{n}, n>3$, using the (quasi)hyperbolic length element

$$
d \sigma=\left(x_{1}^{2}+\ldots+x_{n-1}^{2}\right)^{-1 / 2} d s
$$

and defining as above an $n$-dimensional Jordan domain

$$
D_{n}=\left\{x \in \boldsymbol{R}^{n}:\left|\psi+\frac{\alpha}{\sinh (\sigma)}\right|<\frac{\pi}{2}, \quad 0<\sigma \equiv+\infty\right\}
$$

such that the boundary of $D_{n}$ contains the unit sphere $S^{n-2}$ of the hyperplane $R^{n-1}=$ $\left\{x \in \boldsymbol{R}^{n}: x_{n}=0\right\} \subset \boldsymbol{R}^{n}$. Only the rotations require a somewhat more careful consideration than before. To this purpose we choose antisymmetric linear endomorphisms $A_{1}, \ldots, A_{q}$ of $R^{n-1}$ such that the orbit of

$$
O(t)=\prod_{j=1}^{q} \exp \left(t A_{j}\right) \in S O(n-1) \subset S O(n)
$$

for $t \rightarrow \pm \infty$ is dense in the orthogonal group $S O(n-1)$ of $\boldsymbol{R}^{n-1}$. Let $\tau: D_{n} \rightarrow \boldsymbol{R}$ be the parameter function for $D_{n}$. For all real $\lambda$ we can now define by

$$
f_{\lambda}(x)=O(\lambda \tau(x)) x
$$


a quasiconformal mapping $f_{\lambda}: D_{n} \rightarrow D_{n}$ with

where

$$
K\left(f_{\lambda}\right) \leqq c_{1}(\lambda a)^{n},
$$

$$
a=\sum_{j=1}^{q}\left\|A_{j}\right\| .
$$

Furthermore, for all $b \in S^{n-2} \subset \partial D_{n}$

when only $\lambda \neq 0$.

$$
C\left(f_{\lambda}, b\right)=S^{n-2}
$$

\section{References}

[1] CarathÉodory, C.: Konforme Abbildung Jordanscher Gebiete. - Math. Ann. 73, 1913, 305320.

[2] Courant, R.: Über eine Eigenschaft der Abbildungsfunktionen bei konformer Abbildung. Nachr. Königl. Gesellsch. Wiss. Göttingen Math.-Phys. K1. 1914, 101-109.

[3] FABer, G.: Über den Hauptsatz aus der Theorie der konformen Abbildungen. - Sitzungsber. Math.-Phys. Kl. Bayer. Akad. Wiss. München, 1922, 91-100.

[4] Gehring, F. W., and J. VÄISÄLÄ: The coefficients of quasiconformality of domains in space. Acta Math. 114, 1965, 1-70.

[5] Lehto, O., und K. I. ViRTANen: Quasikonforme Abbildungen. - Springer-Verlag, BerlinHeidelberg-New York, 1965.

[6] OsGood, W. F., and E. H. TAYLOR: Conformal transformations on the boundaries of their regions of definition. - Trans. Amer. Math. Soc. 14, 1913, 277-298.

[7] VÄISÄLÄ, J.: On quasiconformal mappings of a ball. - Ann. Acad. Sci. Fenn. Ser. A I Math. 304, 1961, $1-7$.

[8] VÄISÄLÄ, J.: Lectures on $n$-dimensional quasiconformal mappings. - Lecture Notes in Mathematics 229. Springer-Verlag, Berlin-Heidelberg-New York, 1971.

Institut Mittag-Leffler

Auravägen 17

S-182 62 Djursholm

Sweden
University of Jyväskylä

Department of Mathematics

SF-40100 Jyväskylä

Finland

Received 16 December 1983 